\title{
Magnetostatic Interaction in Fe-Co Nanowires
}

\author{
Laura Elbaile, ${ }^{1}$ Rosario D. Crespo, ${ }^{1}$ Víctor Vega, ${ }^{2}$ and José A. García ${ }^{1}$ \\ ${ }^{1}$ Departamento de Física, Facultad de Ciencias, Universidad de Oviedo, c/ Calvo Sotelo s/n, 33007 Oviedo, Spain \\ ${ }^{2}$ Unidad de Membranas Nanoporosas, SCTs Campus del Cristo, 33006 Oviedo, Spain
}

Correspondence should be addressed to Laura Elbaile, elbaile@uniovi.es

Received 21 March 2012; Revised 23 May 2012; Accepted 23 May 2012

Academic Editor: Zhi Li Xiao

Copyright () 2012 Laura Elbaile et al. This is an open access article distributed under the Creative Commons Attribution License, which permits unrestricted use, distribution, and reproduction in any medium, provided the original work is properly cited.

\begin{abstract}
Arrays of Fe-Co alloy nanowires with diameter around $35 \mathrm{~nm}$ and several micrometers in length have been synthesized by codepositing $\mathrm{Fe}$ and $\mathrm{Co}$ into porous anodic alumina. The morphology, structure, and magnetic properties of the nanowires (hysteresis loops and remanence curves) were characterized by SEM, TEM, X-ray diffraction (XRD), and VSM, respectively. The XRD patterns indicate that the Fe-Co nanowires present a body-centered cubic (bcc) structure and a preferred (110) orientation perpendicular to the template surface. From the hysteresis loops obtained with the magnetic field applied in the axis direction of the nanowires, we can observe that the coercive field slightly decreases when the nanowire length increases. This magnetic behaviour is analyzed considering the shape anisotropy and the dipolar interactions among nanowires.
\end{abstract}

\section{Introduction}

Nanowire arrays are of significant interest from fundamental point of view for studying the magnetic interactions in ordered magnetic systems as well as potential materials for advanced storage and new magnetic devices [1]. During the last years many research efforts concerning the ability to produce high-density nanowire arrays for perpendicular recording media have been made; for this purpose, magnetic nanowires should have high magnetization and relative large coercivity. $\mathrm{Fe}_{x} \mathrm{Co}_{1-x}$ alloys are appropriate materials because of their high saturation magnetization; the highest saturation magnetization; occurs in 65\% iron-35\% cobalt alloys, high Curie temperatures, and low magnetocrystalline anisotropy [2].

Zhan et al. [3] have fabricated arrays of $\mathrm{Fe}_{1-x} \mathrm{Co}_{x}(0.0 \leq$ $x \leq 1.0$ ) nanowires by codepositing $\mathrm{Fe}$ and Co into porous alumina membranes and have investigated the structure and magnetic properties. Other authors [4-6] have investigated the structure and magnetic properties of the $\mathrm{Fe}$ Co nanowires of as-deposited and annealed samples. In the other hand, Fodor et al. [7] have studied the dipolar interaction in Fe-Co arrays of nanowires of the same length in the range from 12 to $48 \mathrm{~nm}$ in diameter. Recently, Lim et al. [8] have investigated the length dependence of the coercivity of $\mathrm{CoFe}_{2}$ nanowire arrays with high length to diameter ratio ranging from 200 to 1000 . The purpose of the present paper is to understand better the role of the wire length on the magnetic properties. To carry out this study, we have selected the nanowire arrays with length to diameter ratio from 60 to 240 of the $\mathrm{Fe}_{69} \mathrm{Co}_{31}$ composition, which presents very low magnetocrystalline anisotropy, and therefore the magnetic properties of nanowire arrays depend basically on the geometrical parameters of the nanowires and on the magnetostatic interactions among them.

\section{Experimental Procedure}

2.1. Fabrication of AAO Membranes and Nanowire Arrays. The ordered porous alumina membranes, with pore diameter about $35 \mathrm{~nm}$ and interpore distance of $105 \mathrm{~nm}$, were prepared from high-purity (99.999\%) aluminium foils via two-step anodization process [9]. This two-step anodization method allows fabricating alumina templates with large-area densities of nanopores which are self ordered following a centred hexagonal arrangement. Although nanopore arrays are almost ideally ordered in a short-range scale, longrange ordering is lost, appearing micrometric size domains of highly ordered nanopores separated by grain boundaries where the ordering is modified. Before the anodization process, the samples were cleaned by sonication in isopropanol 
and ethanol and then electropolished in a mixture of ethanol and perchloric acid (3:1 in vol.) at $20 \mathrm{~V}$ for $5 \mathrm{~min}$ in order to reduce the surface roughness and improve the high-ordering degree of the nanopores in the AAO template. The first anodization process was performed by applying a constant voltage of $40 \mathrm{Vcc}$ and using an oxalic $0.3 \mathrm{M}$ acid solution at $1-3^{\circ} \mathrm{C}$ for $24 \mathrm{~h}$. The second anodization step was performed in the same conditions as the first one for 4 hours, giving an estimated membrane thickness of about $8 \mu \mathrm{m}$. After the second anodization process the alumina barrier layer, which exists at the $\mathrm{Al}_{2} \mathrm{O}_{3} / \mathrm{Al}$ interface, is thinned by stepwise, decreasing the anodizing potential until $4.5 \mathrm{~V}$ [10] in the same conditions as those employed during the anodization processes, $0.3 \mathrm{M}$ oxalic acid solution at $1-3^{\circ} \mathrm{C}$, in order to improve the conductivity of the alumina barrier layer and therefore to enable an homogeneous subsequent deposition process.

The Fe and Co ions were codeposited into the pores of AAO membranes at room temperature by pulsed electrodeposition method $[11,12]$ from an aqueous bath containing $\mathrm{CoSO}_{4} \times 7 \mathrm{H}_{2} \mathrm{O} 0.045 \mathrm{M}, \mathrm{FeSO}_{4} \times 7 \mathrm{H}_{2} \mathrm{O} 0.18 \mathrm{M}$, boric acid $0.54 \mathrm{M}$, ascorbic acid $0.006 \mathrm{M}$, and $\mathrm{NaOH}$ to obtain a $\mathrm{pH}$ about 4 . The pulsed electrodeposition process has consisted in combination of a galvanostatic pulse when the Fe-Co alloy is deposited $\left(25.5 \mathrm{~mA} / \mathrm{cm}^{2}, 8 \mathrm{~ms}\right)$, followed by a potentiostatic one $(+4.5 \mathrm{~V}, 1 \mathrm{~ms})$ when the capacitor formed by the interface $\mathrm{Al} / \mathrm{Al}_{2} \mathrm{O}_{3}$ barrier layer/electrolyte is discharged, and finally a long rest pulse of $0.7 \mathrm{~s}$. Pulsed electrodeposition allows better control than galvanostatic electrodeposition over the deposition parameters, such as deposition rate and ion concentration at the deposition interface, and therefore, it allows an accurate control over the aspect ratio of nanowires by monitoring the total amount of charge passed through the electrochemical cell and makes possible to guarantee the homogeneity of the nanowire composition. The length of wires was controlled by using different deposition times. The deposition times were 30, 75 , and $120 \mathrm{~min}$ for the wires of 2,5 , and $8 \mu \mathrm{m}$ in length, respectively. The obtained samples are hexagonally closepacked arrays of parallel Fe-Co wires being $105 \mathrm{~nm}$ for the interwire distance and $35 \mathrm{~nm}$ for the average diameter. EDX analysis indicates that the atomic ratio of $\mathrm{Fe}$ and $\mathrm{Co}$ in nanowires is close to $69: 31$.

2.2. Morphological and Structural Characterization. XRD pattern of $\mathrm{Fe}_{69} \mathrm{Co}_{31}$ ranging from $30^{\circ}$ to $90^{\circ}$ for the sample of $2 \mu \mathrm{m}$ is shown as an example in Figure 1. In the other samples similar XRD patterns have been observed. The patterns indicate that the deposited nanowires have a bcc structure and a preferred orientation which is perpendicular to the template surface. The inset shows the peaks used for grain size calculation for samples of 2 and $8 \mu \mathrm{m}$. Based on Scherrer formula, the grain size has been estimated to be $16 \pm 2 \mathrm{~nm}$ and $18 \pm 2 \mathrm{~nm}$ for 2 and $8 \mu \mathrm{m}$, respectively.

An estimation of the mean values of nanowire length can be obtained from the SEM images after partial dissolving of the alumina membrane in $1 \mathrm{M} \mathrm{NaOH}$ aqueous solution. As an example, the SEM image for Fe-Co nanowire array of

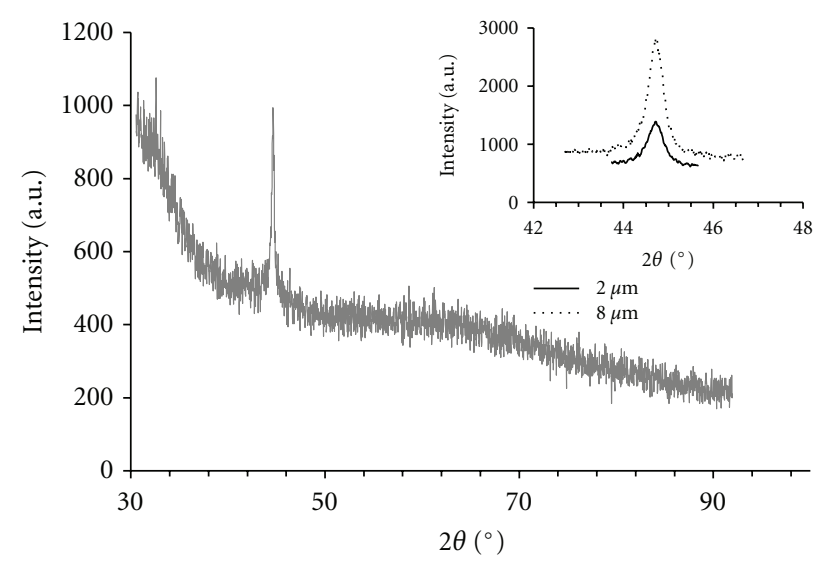

FIGURE 1: XRD patterns of $\mathrm{Fe}_{69} \mathrm{Co}_{31}$ nanowire arrays of $2 \mu \mathrm{m}$. Inset shows maxima used for average grain size calculation.

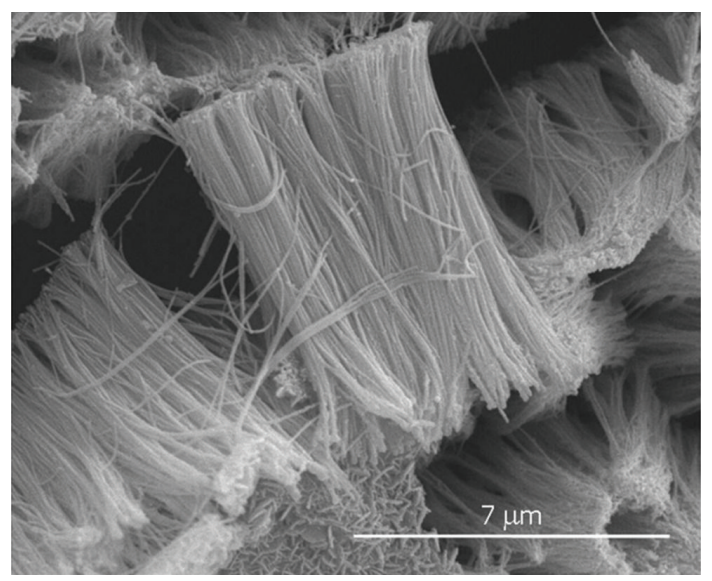

FIGURE 2: SEM image for Fe-Co nanowire array of $8 \mu \mathrm{m}$ length obtained after partial dissolving of the alumina membrane.

about $8 \mu \mathrm{m}$ length is shown in Figure 2. TEM images show that the nanowires are uniform in diameter along their length and the diameter of the wires, in all studied samples, is about $35 \mathrm{~nm}$. Figure 3 is a TEM image of the sample of $8 \mu \mathrm{m}$ in length, which shows the preferred orientation of the closepacked planes almost parallel to the wire axis.

2.3. Magnetic Characterization. The magnetic properties of the Fe-Co nanowire arrays in the alumina template were measured by using a vibrating sample magnetometer (VSM). Hysteresis loops of Fe-Co nanowires were obtained as a function of the magnetic field applied parallel and perpendicular to the longitudinal axis of nanowires. The dominant interactions in these arrays were investigated by analyzing the isothermal remanent magnetization IRM and the dc demagnetization remanence DCD. The IRM curve is measured on previously demagnetized sample by applying a positive field $H$ which is removed and the remanence $M_{r}$ is measured. The process is repeated for different larger fields until saturation is reached. The DCD curve is measured by first saturating the sample in a positive field 


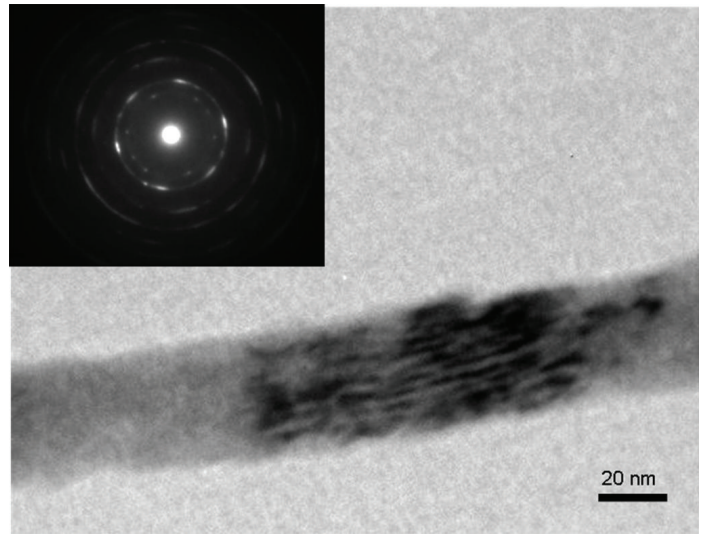

FIGURE 3: TEM image for nanowire of $8 \mu \mathrm{m}$ length showing the preferred orientation of the close packed planes, which are almost parallel to the wire axis. In the inset selected area diffraction pattern corresponding to the micrograph is presented.

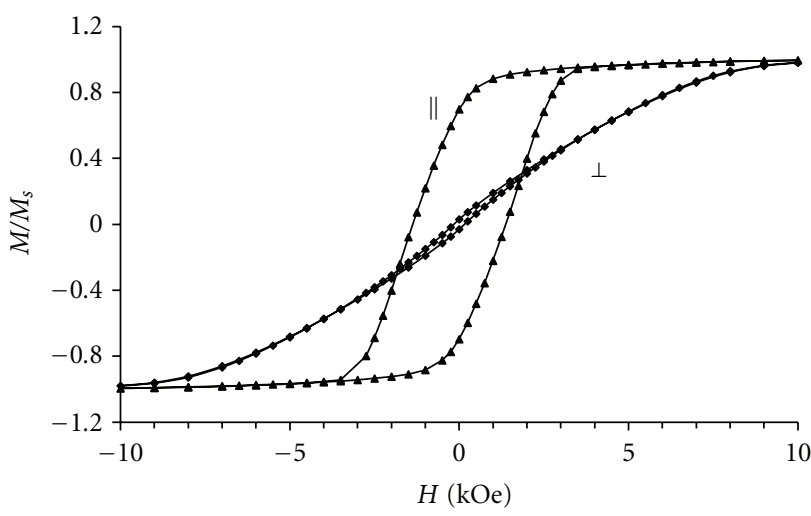

FIGURE 4: Normalized hysteresis loops of Fe-Co nanowire array with $5 \mu \mathrm{m}$ in length. The symbols $\|$ and $\perp$ means that applied magnetic field is parallel and perpendicular to the wire axes, respectively.

and then measuring the remanence $M_{d}$ after application of progressively larger negative fields. The two sets of remanent magnetization data are used to evaluate

$$
\delta M=\frac{M_{d}(H)}{M_{r}(\infty)}-\left[1-2 \frac{M_{r}(H)}{M_{r}(\infty)}\right],
$$

where the values for $M_{d}$ and $M_{r}$ are normalized with respect to the isothermal remanence for high fields. $\delta M$ plots are widely used in the characterization of interactions in magnetic materials and give a global value for the overall interactions of the sample. The concept of $\delta M$ derives from the Wohlfarth relation [13] which shows that the values of remanence for the magnetizing and demagnetizing are the same for a noninteracting system and the differences arise due to interactions.

\section{Results and Discussion}

Figure 4 shows, as an example, the normalized hysteresis loops for $\mathrm{Fe}_{69} \mathrm{Co}_{31}$ nanowires of $5 \mu \mathrm{m}$ in length when the applied magnetic field is parallel and perpendicular to

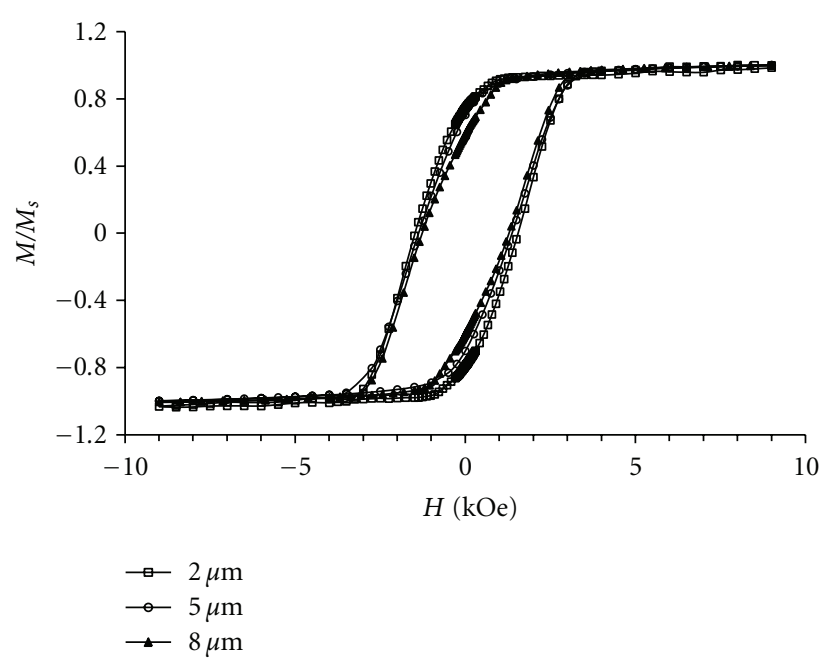

FIGURE 5: Normalized hysteresis loops for Fe-Co nanowire arrays of 2, 5 and $8 \mu \mathrm{m}$ in length measured with the applied field parallel to the wire axes.

TABLE 1: Coercive fields and squareness with the applied magnetic field out of plane of the samples.

\begin{tabular}{lcc}
\hline Wire length $(\mu \mathrm{m})$ & $\mathrm{Hc}(\mathrm{Oe})$ & $\mathrm{Mr} / \mathrm{Ms}$ \\
\hline 2 & 1520 & 0.76 \\
5 & 1400 & 0.70 \\
8 & 1270 & 0.58 \\
\hline
\end{tabular}

the nanowires. These hysteresis loops reveal that the array exhibits uniaxial magnetic anisotropy with the easy axis parallel to the nanowires. In the other samples the same magnetic behavior has been observed.

Figure 5 illustrates the normalized hysteresis loops for the samples with lengths 2,5 , and $8 \mu \mathrm{m}$ measured with the applied field along the axis of the wires. The values of the coercivity $H_{c}$, and squareness $M_{r} / M_{s}$ for all studied samples, with $M_{s}$ being the saturation magnetization, are shown in Table 1. From these results a decreasing of the coercive field and the remanence when the length increases can be observed. When the applied magnetic field is perpendicular to the axis of the wire, all the samples present a similar behavior being the saturation field about $9 \sim 10 \mathrm{kOe}$.

In Figure 6 we show the $\delta M$ plots for Fe-Co samples of 2 and $8 \mu \mathrm{m}$ in length derived from dc demagnetization remanent $M_{d}(H)$ and isothermal remanent $M_{r}(H)$ normalized with respect to the isothermal remanence $M_{r}(\infty)$ for high fields. The plots show negative peaks for the investigated samples. This fact reveals that the predominant interaction is magnetostatic $[7,14]$. From the $\delta M$ curves only slight differences between the amplitude of the peaks for both samples indicating a similar magnitude of magnetostatic interaction for both samples can also be observed; nevertheless, the peak for the sample of $8 \mu \mathrm{m}$ in length is shifted at $H / H_{c}$ value bigger than 1 .

To achieve better understanding of the magnetic behaviour of nanowires, the switching field distribution 


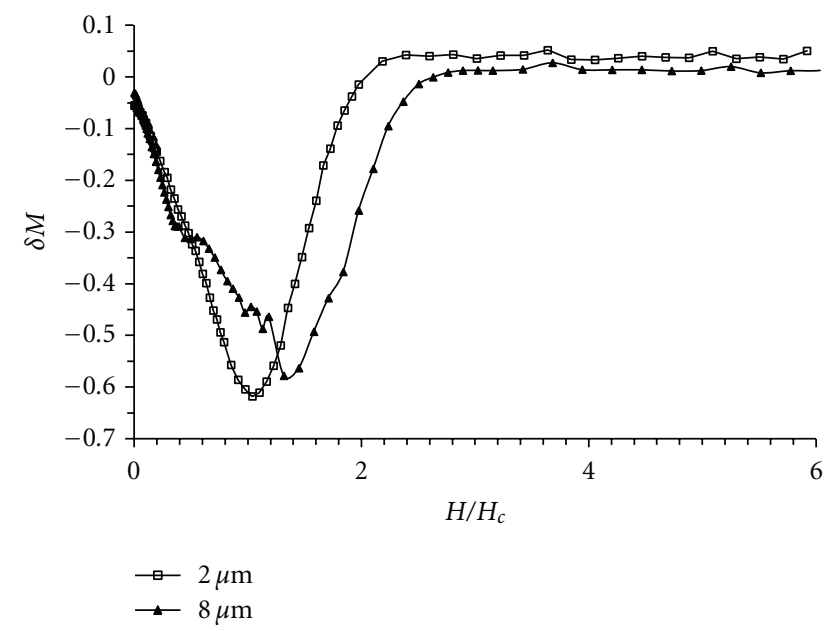

Figure 6: Modified Henkel plots for Fe-Co nanowire arrays of 2 and $8 \mu \mathrm{m}$ length. The zero magnetization state is obtained by ac demagnetization with the applied magnetic field parallel to the nanowires.

(SFD) associated with reversal magnetization has been calculated as field derivatives of $M_{r}(H)$ and $M_{d}(H)$

$$
\mathrm{SFD}=\left|\frac{d\left(M_{r} / M_{r, \max }\right)}{d H}\right|
$$

which gives information about the magnetization and demagnetization processes. For the IRM case, measured from a demagnetized state, the SFD measures the distribution of energy barriers to wall motion for both samples. For the DCD case, where the process starts when the samples are saturated, the SFD measures the energy barrier to domain nucleation [15].

The SFDs corresponding to the magnetization process from the demagnetized state and demagnetization process for samples of $2 \mu \mathrm{m}$ and $8 \mu \mathrm{m}$ in length are shown in Figure 7. As can be seen, the SFDs obtained from the two remanence curves are different due to the different initial configuration of the magnetization and evidence that is harder to magnetize a demagnetized sample than a previously magnetized one. For the sample of $2 \mu \mathrm{m}$, starting from the demagnetized state, few walls move until a field $\cong 200$ Oe has been applied and reach their maximum at about $1.90 \mathrm{kOe}$, a value which coincides within $5 \%$ with the applied field for the peak of the SFD for the IRM. The values of full width at half maximum are very different for the two SFDs indicating the magnetostatic interaction. The SFD for the IRM curve of the sample $8 \mu \mathrm{m}$ in length shows a broad irregular distribution with various peaks and from the SFD for DCD we appreciate that the demagnetization starts from null fields which suggest the presence of strong local demagnetizing fields at remanence.

In the studied wires, the out-of-plane demagnetizing factor as well as the magnetocrystalline anisotropy constant are nearly negligible; so, in order to interpret the sheared hysteresis loops and the dependence of coercivity on wire
TABLE 2: Values of $H_{\mathrm{mp}}$ and $H_{\mathrm{av}}$ for an array of $9 \times 10^{6}$ wires.

\begin{tabular}{lcccc}
\hline Length $(\mu \mathrm{m})$ & \multicolumn{2}{c}{$H_{\mathrm{mp}}(\mathrm{kOe})$} & \multicolumn{2}{c}{$H_{\mathrm{av}}(\mathrm{kOe})$} \\
& $\begin{array}{c}\text { Magnetic } \\
\text { charges }\end{array}$ & Dipoles & $\begin{array}{c}\text { Magnetic } \\
\text { charges }\end{array}$ & Dipoles \\
\hline 2 & 2.30 & 2.30 & 2.23 & 2.23 \\
5 & 2.28 & 2.28 & 2.25 & 2.25 \\
8 & 2.27 & 2.26 & 2.24 & 2.24 \\
\hline
\end{tabular}

length, the magnetostatic field in the middle point $H_{\mathrm{mp}}$ of the central nanowire and the average field interaction $H_{\mathrm{av}}$ in the central nanowire, created by the array of magnetically saturated wires out of plane, have been evaluated for all the samples: (a) treating each wire as a linear chain of several thousand point dipoles [16] and (b) considering the axially magnetized nanowire like a pair of magnetic monopoles located at its ends. To do that, first, the magnetic stray field generated by a single-magnetized nanowire in the neighboring region has been determined. Then, the sum of the stray field produced by all the wires on a specific wire at the center of the array has been evaluated.

As can be seen in Figure 8, which shows the evolution of $H_{\mathrm{mp}}$ and $H_{\mathrm{av}}$ for the out-of-plane magnetized sample of $8 \mu \mathrm{m}$ in length with the number of wires, the dipolar field results strongly dependent on the array size and a large number of wires are required for reaching convergence. So, numerical calculations were performed with a maximum of $9 \times 10^{6}$ wires distributed in the nodes of a hexagonal-close-packed lattice.

In Table 2, the values of $H_{\mathrm{mp}}$ and $H_{\mathrm{av}}$ for an array of $9 \times 10^{6}$ wires of the studied samples are presented. From this table, we can only notice slight differences in the average interaction field values between them. We can also observe that the evaluated values coincide using both dipole and magnetic monopoles models in all the samples.

The magnetic properties of $\mathrm{Fe}, \mathrm{Ni}$, and their alloy nanowire arrays, electrodeposited into the nanopores of AAO membranes, which present a small magnetocrystalline anisotropy, depend on the aspect ratio of nanowires and the dipolar interaction between them due to high-pore densities. In these nanowires, the magnetization lies along the wire axis and present high coercivity and squarenes [17]. On the other hand, the cobalt nanowires lead to a very wide range of magnetic properties as the magnetocrystalline anisotropy $\sim 10^{4} \mathrm{~J} / \mathrm{m}^{3}$ competes to the shape anisotropy [18].

In our case the dependence of the coercivity and squareness on the length cannot be explained neither by the shape anisotropy nor by the crystalline structure. These results could be due to that the length to diameter ratio in the nanowires of the present work is not very high, and therefore other factors could affect their magnetic behaviour. The broad distribution of the SFD for the IRM curve of the $8 \mu \mathrm{m}$ in length sample suggests a nonuniform filling of nanopores which would explain the abnormal behaviour of coercivity and squareness. This lack of nanowire length uniformity has been confirmed by SEM analysis. 


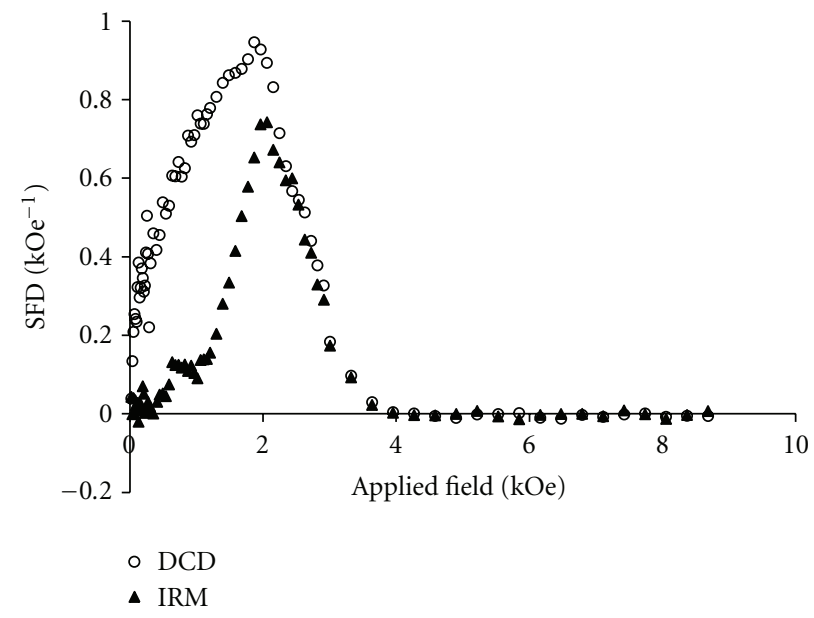

(a)

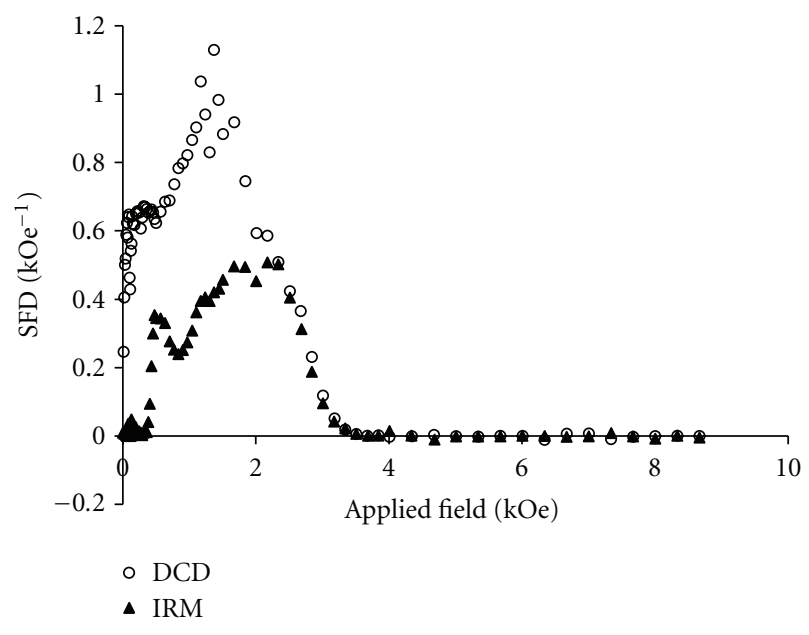

(b)

FIGURE 7: Switching field distributions calculated from the DCD and IRM remanence curves (a) for the sample of $2 \mu \mathrm{m}$ and (b) for the sample $8 \mu \mathrm{m}$.

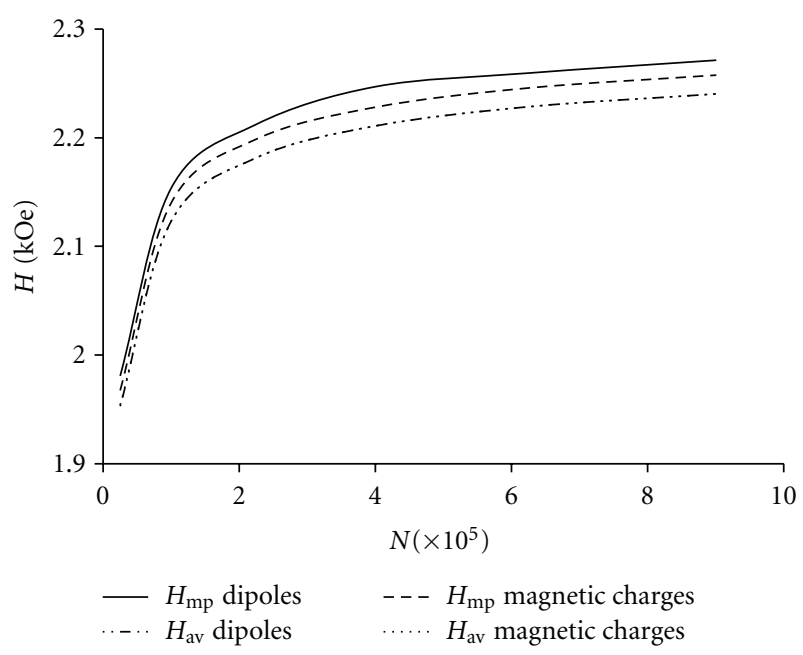

Figure 8: Evolution of $H_{\mathrm{mp}}$ and $H_{\mathrm{av}}$ for the out-of-planemagnetized sample of $8 \mu \mathrm{m}$ in length with different number of wires $N$.

\section{Conclusions}

The experimental results show a monotonic decreasing of coercivity and squareness in relation to the length of the wires.

The magnetostatic field, parallel to the wire axes, was modeled taking into account the magnetic charges at the ends of the wires as well as treating the wire like a linear chain of several thousand point dipoles. Slight differences between the calculated values from the two models have been noticed.

The dipolar field results strongly dependent on the size of the array and a large number of wires $>10^{6}$ that corresponds to a sample of $\cong 0.1 \mathrm{~mm}^{2}$ is required for reaching convergence. Numerical calculation has been performed with a maximum of $9 \times 10^{6}$ wires. A weak influence of the length on the magnetostatic interaction among nanowires is observed.

The broad distribution of the SFD for the IRM curves of the $8 \mu \mathrm{m}$ in length sample suggests a nonuniform filling of nanopores which would explain the abnormal behaviour of coercivity and squareness.

\section{Acknowledgments}

This work was supported in part by the MEC of Spain through Grant ref. MAT2008-06784 and by the FICYT of Principado de Asturias (ref. IB09-128). The authors are grateful to V. de la Prida, C. Alvarez, A. Quintana, and D. Martinez in charge of Nanoporous Membranes, TEM, SEM, and XRD scientific services of the University of Oviedo respectively, and to Dr. I. Orue responsible of Magnetic Measurement Service of the University of the Basque Country.

\section{References}

[1] R. P. Cowburn and M. E. Welland, "Room temperature magnetic quantum cellular automata," Science, vol. 287, no. 5457, pp. 1466-1468, 2000.

[2] D. Jiles, Introduction to Magnetism and Magnetic Materials, Chapman and Hall, London, UK, 1991.

[3] Q. Zhan, Z. Chen, D. Xue et al., "Structure and magnetic properties of $\mathrm{Fe}-\mathrm{Co}$ nanowires in self-assembled arrays," Physical Review B, vol. 66, no. 13, Article ID 134436, 6 pages, 2002.

[4] D. H. Qin, L. Cao, Q. Y. Sun, Y. Huang, and H. L. Li, "Fine magnetic properties obtained in FeCo alloy nanowire arrays," Chemical Physics Letters, vol. 358, no. 5-6, pp. 484-488, 2002.

[5] Y. Guo, D. H. Qin, J. B. Ding, and H. L. Li, "Annealing and morphology effects on the $\mathrm{Fe}_{0.39} \mathrm{Co}_{0.61}$ nanowire arrays," Applied Surface Science, vol. 218, no. 1-4, pp. 106-112, 2003.

[6] H. L. Su, G. B. Ji, S. L. Tang, Z. Li, B. X. Gu, and Y. W. Du, "Geometry dependence of the annealing effect 
on the magnetic properties of $\mathrm{Fe}_{48} \mathrm{Co}_{52}$ nanowire arrays," Nanotechnology, vol. 16, no. 4, pp. 429-432, 2005.

[7] P. S. Fodor, G. M. Tsoi, and L. E. Wenger, "Investigation of magnetic interactions in large arrays of magnetic nanowires," Journal of Applied Physics, vol. 103, no. 7, Article ID 07B713, 2008.

[8] S. L. Lim, F. Xu, N. N. Phuoc, and C. K. Ong, "Length dependence of coercivity in $\mathrm{CoFe}_{2}$ nanowire arrays with high aspect ratios," Journal of Alloys and Compounds, vol. 505, no. 2, pp. 609-612, 2010.

[9] H. Masuda and K. Fukuda, "Ordered metal nanohole arrays made by a two-step replication of honeycomb structures of anodic alumina," Science, vol. 268, no. 5216, pp. 1466-1468, 1995.

[10] R. C. Furneaux, W. R. Rigby, and A. P. Davidson, "The formation of controlled-porosity membranes from anodically oxidized aluminium," Nature, vol. 337, no. 6203, pp. 147-149, 1989.

[11] K. Nielsch, F. Müller, A. P. Li, and U. Gösele, "Uniform nickel deposition into ordered alumina pores by pulsed electrodeposition," Advanced Materials, vol. 12, p. 582, 2000.

[12] V. M. Prida, K. R. Pirota, D. Navas, A. Asenjo, M. HernándezVélez, and M. Vázquez, "Self-organized magnetic nanowire arrays based on alumina and titania templates," Journal of Nanoscience and Nanotechnology, vol. 7, no. 1, pp. 272-285, 2007.

[13] E. P. Wohlfarth, "Relations between different modes of acquisition of the remanent magnetization of ferromagnetic particles," Journal of Applied Physics, vol. 29, no. 3, pp. 595596, 1958.

[14] J. Rivas, A. Kazadi Mukenga Bantu, G. Zaragoza, M. C. Blanco, and M. A. López-Quintela, "Preparation and magnetic behavior of arrays of electrodeposited Co nanowires," Journal of Magnetism and Magnetic Materials, vol. 249, no. 1-2, pp. 220-227, 2002.

[15] D. M. Donnet, V. G. Lewis, J. N. Chapman, K. O’Grady, and H. W. van Kesteren, "Microstructure and hysteresis in $\mathrm{Co} / \mathrm{Pt}$ multilayers," Journal of Physics D, vol. 26, no. 10, pp. 1741$1745,1993$.

[16] L. Clime, P. Ciureanu, and A. Yelon, "Magnetostatic interactions in dense nanowire arrays," Journal of Magnetism and Magnetic Materials, vol. 297, no. 1, pp. 60-70, 2006.

[17] L. Sun, Y. Hao, C. L. Chien, P. C. Searson, and P. C. Searson, "Tuning the properties of magnetic nanowires," IBM Journal of Research and Development, vol. 49, no. 1, pp. 79-102, 2005.

[18] D. J. Sellmyer, M. Zheng, and R. Skomski, "Magnetism of FE, CO and NI nanowires in self-assembled arrays," Journal of Physics Condensed Matter, vol. 13, no. 25, pp. R433-R460, 2001. 

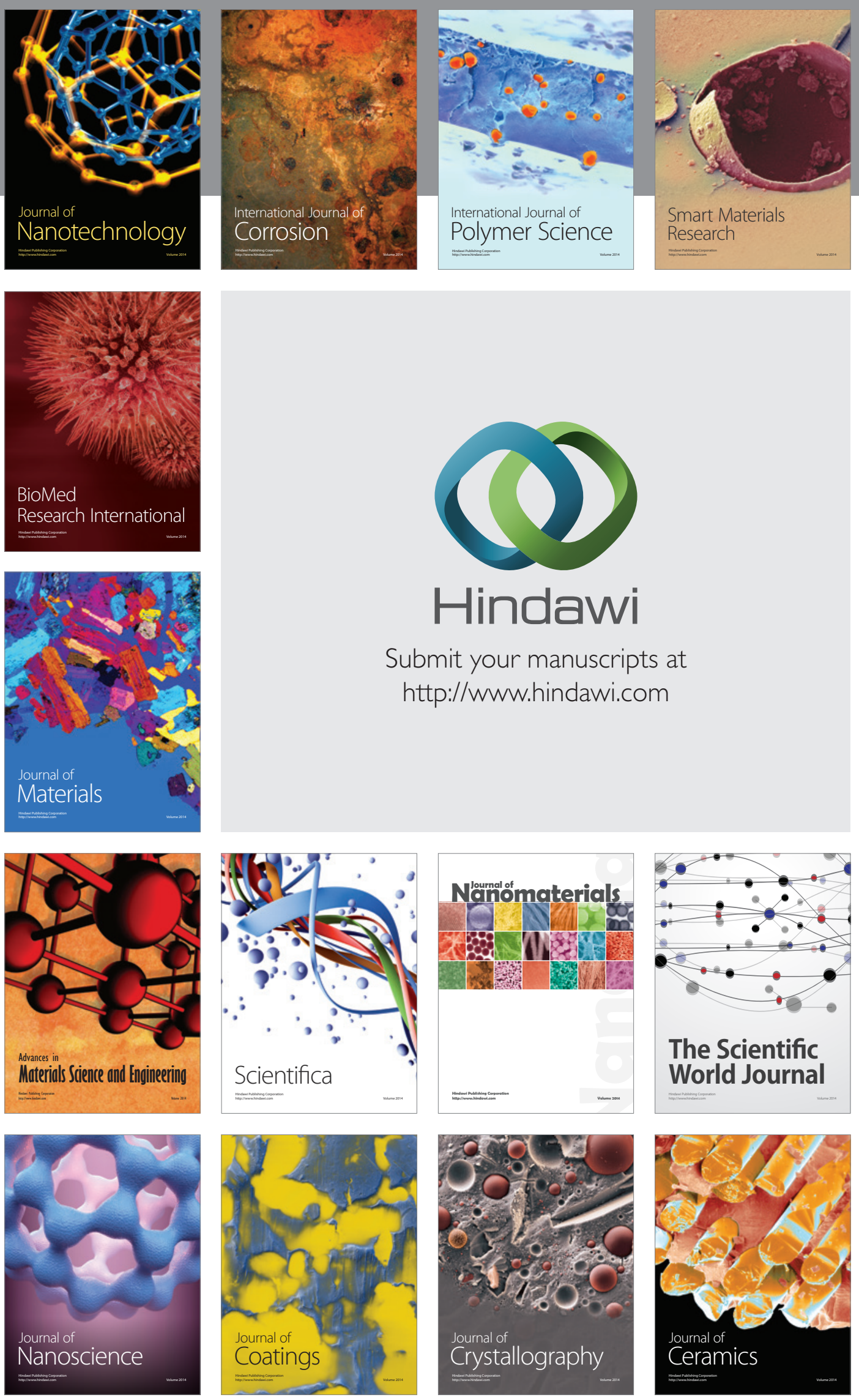

The Scientific World Journal

Submit your manuscripts at

http://www.hindawi.com

\section{World Journal}

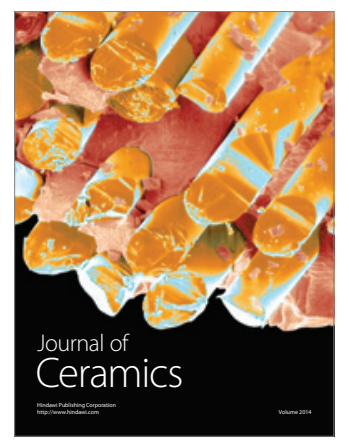

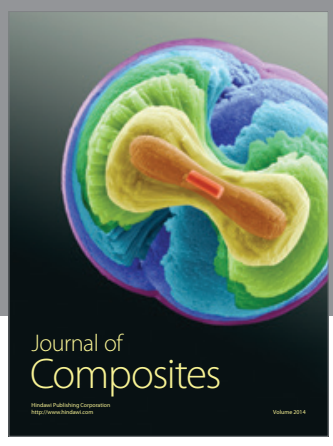
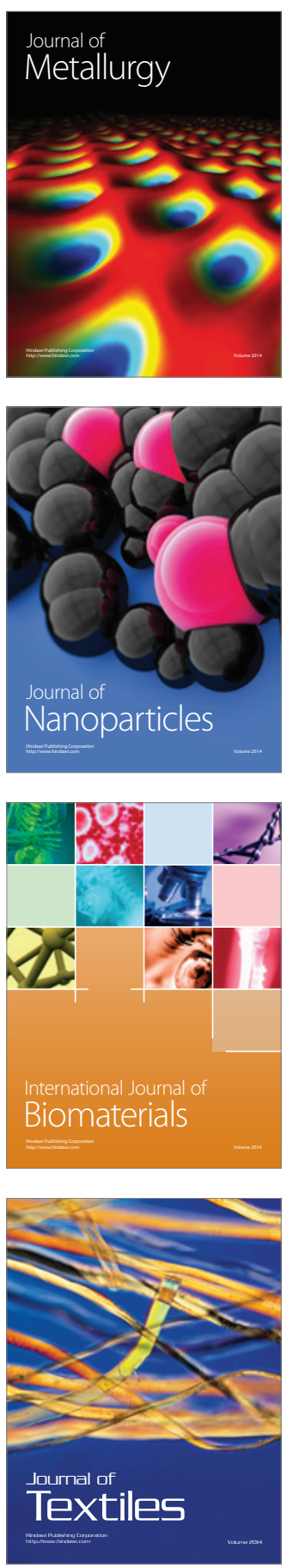